- Orofacial pain usually has a local cause.

- Dental caries and sequelae are the main causes.

- A careful history is crucial to the diagnosis.

- Lancinating pain is typical of trigeminal neuralgia.

- Chronic pain in the absence of organic causes, may be psychogenic.

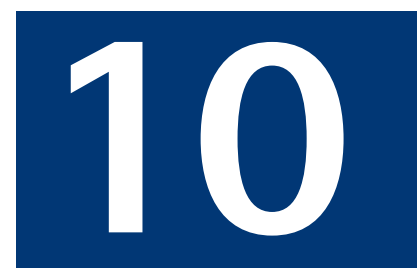

\title{
Oral Medicine - Update for the dental practitioner Orofacial pain
}

\author{
C. Scully ${ }^{1}$ and D. H. Felix ${ }^{2}$
}

This series provides an overview of current thinking in the more relevant areas of oral medicine for primary care practitioners, written by the authors while they were holding the Presidencies of the European Association for Oral Medicine and the British Society for Oral Medicine, respectively. A book containing additional material will be published. The series gives the detail necessary to assist the primary dental clinical team caring for patients with oral complaints that may be seen in general dental practice. Space precludes inclusion of illustrations of uncommon or rare disorders, or discussion of disorders affecting the hard tissues. Approaching the subject mainly by the symptomatic approach - as it largely relates to the presenting complaint - was considered to be a more helpful approach for GDPs rather than taking a diagnostic category approach. The clinical aspects of the relevant disorders are discussed, including a brief overview of the aetiology, detail on the clinical features and how the diagnosis is made. Guidance on management and when to refer is also provided, along with relevant websites which offer further detail.

\section{ORAL MEDICINE \\ 1. Aphthous and other common ulcers \\ 2. Mouth ulcers of more serious connotation \\ 3. Dry mouth and disorders of salivation \\ 4. Oral malodour \\ 5. Oral white patches \\ 6. Oral red and hyperpigmented patches \\ 7. Orofacial sensation and movement \\ 8. Orofacial swellings and lumps \\ 9. Oral cancer \\ 10. Orofacial pain}

1*Professor, Consultant, Dean, Eastman Dental Institute for Oral Health Care Sciences, 256 Gray's Inn Road, UCL,

University of London, London WC1X 8LD

${ }^{2}$ Consultant, Senior Lecturer, Glasgow Dental Hospital and School, 378

Sauchiehall Street, Glasgow G2 3JZ I

Associate Dean for Postgraduate Dental Education, NHS Education for Scotland, 2nd Floor, Hanover Buildings, 66 Rose Street, Edinburgh EH2 2NN

${ }^{*}$ Correspondence to: Professor Crispian Scully CBE

Email:c.scully@eastman.ucl.ac.uk

\section{Refereed Paper}

(c) British Dental Journal 2006; 200 :

75-80

\section{PAIN}

Pain in the teeth, mouth, face or head usually has a local cause, often the sequelae of dental caries (odontogenic pain). However, psychogenic, neurological and vascular conditions, and conditions where pain is referred from elsewhere, may be responsible (Table 1).

Dental staff will be well versed in pain of local causes and therefore this article discusses mainly the conditions in which specialist help may be indicated. Many of the conditions discussed in previous articles in this series may cause pain.

The real significance to the patient of orofacial pain apart from the pain itself, can range from the benign to potentially lethal conditions. Some orofacial pain or headaches have an obvious but relatively unimportant cause (eg a hangover - caused mainly by the acetaldehyde resulting from metabolism of alcohol); others types of pain have no obvious underlying organic pathology (and are thus termed medically unexplained symptoms [MUS], eg atypical facial pain); some can threaten important faculties such as sight (eg giant cell arteritis), or even life (eg brain tumours).

\section{DIAGNOSIS OF OROFACIAL PAIN}

The history is the most important means of diagnosing orofacial pain (Fig. 1).

In order to differentiate the widely disparate causes, it is essential to determine key points about the pain, especially:

- Location. Valuable information can be obtained by asking if the pain is localised or

\section{Table 1 Causes of orofacial pain}

Local disorders

- teeth and supporting tissues

- jaws

- maxillary antrum

- salivary glands

- pharynx

- eyes

Neurological disorders

- Idiopathic trigeminal neuralgia

- Malignant neoplasms involving the trigeminal nerve

- Glossopharyngeal neuralgia

- Herpes zoster (including post-herpetic neuralgia)

- Multiple sclerosis

Possible psychogenic causes

- Atypical facial pain

- Burning mouth syndrome

- Temporomandibular pain-dysfunction

Vascular disorders

- Migraine

- Migrainous neuralgia

- Giant cell arteritis

Referred pain

- Nasopharyngeal

- Ocular

- Aural

- Cardiorespiratory Angina Lesions in the neck or chest (including lung cancer) 


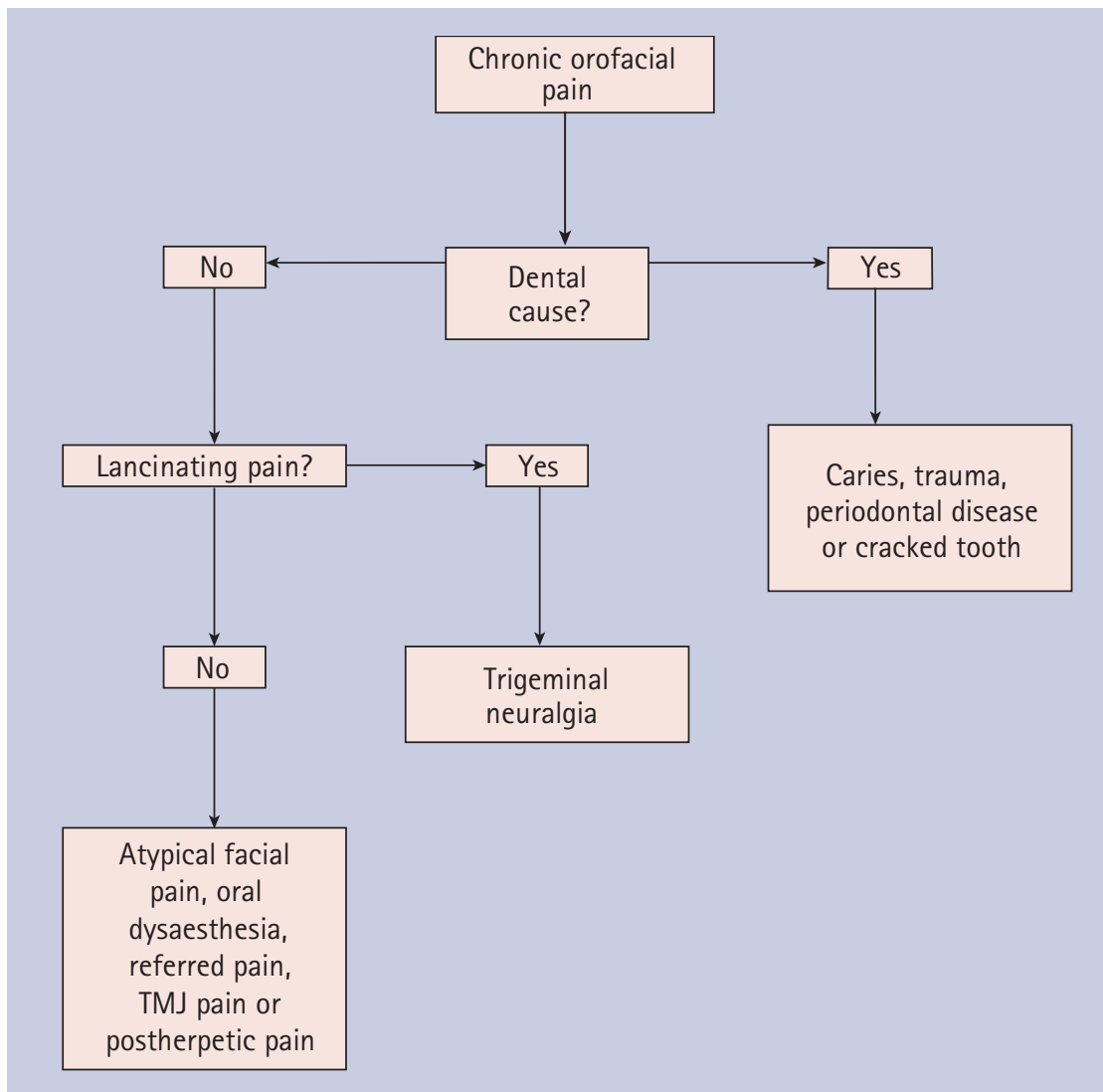

Fig.1 Chronic orofacial pain diffuse, and watching the patient's reaction. For example, patients frequently point with one finger when describing pain of dental causes or trigeminal neuralgia, but atypical facial pain is much more diffuse, and may radiate across the midline.

- Character. Patients should be asked about the severity and character of the pain ie whether the pain is 'sharp', 'dull', 'aching', 'throbbing' or 'shooting'. However, bear in mind that patients often have difficulty finding adequate descriptors. Ask the patient to rate the pain severity on a scale of zero (no pain) to 10 (most severe pain that the patient has experienced), or ask them to mark this on a line divided into 10 equal sections (visual analogue scale) or use an assessment instrument such as the McGill Pain Questionnaire. These 'tools' help assess the severity of pain, accepting always that it is subjective, and they may also be useful in monitoring the response to treatment. Disturbance of the normal sleep pattern by pain is also useful in assessing the severity.

- Duration. The average duration of each episode may help diagnosis. For example, pain from exposed dentine is fairly transient (lasting only for seconds) while the pain from pulpitis lasts for a much longer period. Trigeminal neuralgia is a brief lancinating pain lasting up to about five seconds, although some patients report a persistent background less severe pain - more of a dull ache; migrainous neuralgia typically lasts 30 to 45 minutes, while atypical facial pain is typically persistent.

- Frequency and periodicity. Determine whether the pain occurs at specific times or related to specific events. A 'pain diary' can help. For example, the pain of temporomandibular pain dysfunction syndrome may be more severe on waking if this is associated with nocturnal parafunctional activity such as clenching or tooth grinding. The pain of sinusitis is often aggravated by lying down. Periodic migrainous neuralgia frequently disturbs the patient's sleep at a specific time each night, around 2am. One patient seen by the authors complained of pain fairly typical of periodic migrainous neuralgia, yet appearing around 2pm; it turned out he was a longdistance night driver, sleeping mainly during the day!

- Precipitating, aggravating and relieving factors. It may be necessary to resort to leading questions to ask if temperature, biting, posture, analgesics, alcohol etc affect the pain. For example, heat often aggravates dental pain; touching a trigger zone may precipitate trigeminal neuralgia attacks, stress may worsen atypical facial pain, and alcohol may induce episodes of migrainous neuralgia.

- Associated features. Some types of pain may be associated with other features which are helpful diagnostically, such as the swollen face in dental abscess, nausea and vomiting in migraine, or nasal stuffiness or lacrimation in migrainous neuralgia.

The cause of most orofacial pain is established mainly from the history, and examination findings are also helpful, not least in excluding local pathology. However, it is important to consider the usefulness of a specialist who can arrange additional investigations, particularly imaging of the head and neck, using CT or MRI. It is crucial not to miss detecting organic disease and thus mislabelling the patient as having psychogenic pain, and not to miss a brain tumour underlying a patient with supposed 'idiopathic' trigeminal neuralgia.

\section{LOCAL CAUSES OF OROFACIAL PAIN \\ Odontogenic pain}

Most orofacial pain is of course, related to dental disease - odontogenic causes - and will not be described further.

\section{Mucosal pain}

Pain from oral mucosal lesions can be either localised or diffuse. Localised pain is usually associated with a mucosal break, either an erosion (a partial thickness loss of epithelium) or ulcer (a full thickness loss of epithelium). Of course, the distinction between these painful conditions can at times be difficult or impossible and many patients have both.

Diffuse pain may also be caused by infection, or a systemic underlying deficiency state or other factors, and is usually then described as 'soreness' or sometimes 'burning'.

Mucosal pain may be aggravated by sour, acidic, spicy, or salty foods, so that few affected patients can tolerate or enjoy citrus fruits or 
tomatoes for example. The area is usually also tender to touch.

\section{Other local causes of orofacial pain}

Pain from the jaws can be caused by infection, direct trauma, malignancies, and rarely by Paget's disease. However, unless associated with infection or jaw fracture, retained roots and impacted teeth, and lesions such as cysts, are usually painless.

Malignant tumours usually produce deep, boring pain, sometimes associated with paraesthesia or anaesthesia but odontogenic and other benign tumours of the bone do not normally produce pain. Lip numbness or tingling, therefore, may herald a tumour in the jaw bone.

Pain from salivary gland disorders is mainly caused by duct obstruction, sometimes by infection or a tumour. The pain is usually localised to the affected gland, may be quite severe, and may be intensified by increased saliva production such as before and with meals. Examination may reveal a swollen salivary gland sometimes with tenderness and/or a degree of trismus.

Diseases of the paranasal sinuses and nasopharynx which can cause oral and/or facial pain include sinusitis and tumours - which can remain undetected until they have reached an advanced stage. Any suggestion of a discharge from the nose, or obstruction to breathing, cheek swelling or numbness or tingling of the lip should be taken seriously as they may herald an antral carcinoma.

On occasions if there is dehiscence of the mental nerve, as a result of resorption of the alveolar ridge, pain is caused by pressure from a denture.

\section{TEMPOROMANDIBULAR JOINT PAIN}

Pain from the TMJ may result from dysfunction, trauma, inflammation, and very rarely tumours - either in the head and neck, or even lungs.

\section{Temporomandibular pain-dysfunction syndrome}

Temporomandibular pain-dysfunction syndrome is a very common problem, characterised by pain, clicking and jaw locking or limitation of opening of the jaw. Afflicting young women mainly, factors which have been implicated include over-opening of the mouth, muscle overactivity (eg bruxism, clenching), TMJ disruption and psychiatric history (eg anxiety, stressful life events). Precipitating factors may include local trauma, wide mouth opening, or emotional upset.

\section{Diagnosis}

Diagnosis is clinical. Pain from TMJ disease is usually dull, poorly localised, may radiate widely, is usually intensified by movement of the mandible and may be associated with trismus because of spasm in the masticatory muscles.

Examination may reveal a click from the joint, limited jaw movements, and tender masticatory muscles. Any suggestion of a swollen and/or warm joint, suggests true arthritis.

\section{Management}

Most patients recover spontaneously and progression to arthritis is virtually unknown. Therefore reassurance and conservative measures are the main management. TMJ pain-dysfunction can usually be effectively managed in general practice.

Practitioners are usually well versed with this problem but possible options for treatment in a primary care environment are summarised in the Key points box (see right) and patient guidance in Table 2 .

Recalcitrant cases may need specialist attention, particularly if simple measures fail.

\section{Websites and patient information}

http://www.aaop.org

http://www.tmjd.com/

\section{NEUROLOGICAL (NEUROPATHIC) CAUSES OF OROFACIAL PAIN}

Sensory innervation of the mouth, face and scalp depends on the trigeminal nerve, so that diseases affecting this nerve anywhere in the course from orofacial region to brain, can cause orofacial pain or indeed sensory loss - sometimes with serious implications.

\section{Table 2 Steps to manage TMJ pain dysfunction}

Rest yourself and your jaw

Relax and practice stress reduction

Exercise regularly

Eat soft foods and avoid hard, crusty foods like nuts or hard bread or those that need chewing a great deal

Chew on your back teeth, not the front ones

Eat small bites

Sleep on your side

Avoid joint or muscle damage by avoiding:

- contact sports; wear a mouthguard if you must play contact sports

- excessive jaw use in yawning, grinding and clenching

- chewing gum

- habits such as biting finger nails, pens and pencils or lip

- excessive mouth-opening in long dental appointments

- general anaesthesia

- cradling the telephone between head and shoulder

- wind instrument playing

Reduce muscle pain with analgesics and by applying:

- cold packs for 10 minutes every three hours for 72 hours after injury

- hot packs for 20 minutes every three hours to uninjured joints/muscles

Re-educate the jaw opening:

Open your mouth with a hinge movement: exercise your jaw twice daily, opening five times in front of a mirror, ensuring the jaw opens vertically downwards without deviating sideways

Exercise your jaw three times daily for five timed minutes:

- close your mouth on the back teeth

- put the tip of your tongue on the palate behind your front teeth

- move the tongue back across the palate as far as it will go

- keep the tongue in this position with the teeth closed for 10 seconds

- open your mouth slowly until the tongue starts to leave the palate

- keep that position for 10 seconds

- close your mouth

- repeat over five minutes
Key points for dentists:

Management of TMJ

pain-dysfunction

- Reassurance/explanation of the benign and self- limiting nature o the problem

- Rest (eg soft diet and limitation of movement)

- Anti-inflammatory analgesic (eg buprofen $400 \mathrm{mg}$ three times a day)

- Occlusal splint therapy

- Local physiotherapy

Key points for patients: Temporomandibular (TMJ) paindysfunction

- This is a common condition

- It appears to be related to stress, joint damage or habits involving the teeth and joints (eg tooth clenching or grinding)

- There are no serious long-term consequences; arthritis does not result

- The symptoms usually clear spontaneously after some months but meantime, rest, exercises, splints, or drugs may help. 
Fig. 2 Herpes zoster, palate

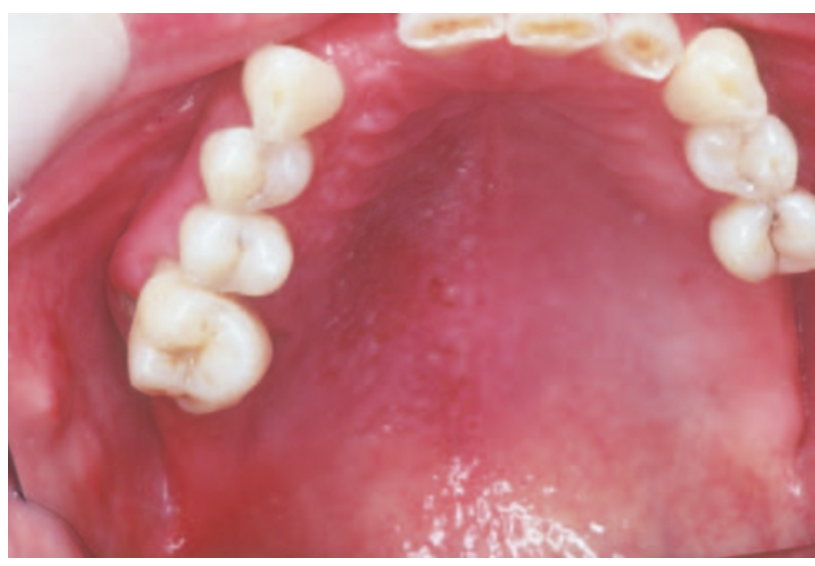

Such causes include:

- trauma

- cerebrovascular disease

- demyelinating disease (eg multiple sclerosis)

- neoplasia (eg nasopharyngeal, antral or brain tumours); (or infections such as herpes zoster or HIV/AIDS (Fig. 2).

\section{Idiopathic trigeminal neuralgia}

Idiopathic trigeminal neuralgia (ITN) is an uncommon nerve disorder that causes episodes of unilateral intense, stabbing, electric shocklike pain in the areas of the face where the branches of the nerve are distributed - lips, eyes, nose, scalp, forehead, upper jaw, or lower jaw. ITN onset is mainly in the 50-70 year age group.

The cause of ITN is unclear, but one hypothesis is that a cerebral blood vessel becomes atherosclerotic and therefore less flexible with age, then pressing on the roots of the trigeminal nerve in the posterior cranial fossa - causing neuronal discharge.

The characteristic features of ITN are summarised as:

- Paroxysmal attacks of facial or frontal pain which lasts a few seconds to less than two minutes. These attacks occur especially in the morning, and rarely cause sleep disturbance.

- Pain has at least four of the following characteristics:

- Distribution along one or more division of the trigeminal nerve.

- Sudden intense, sharp superficial, stabbing or burning in quality.

- Pain intensity severe.

- Precipitation from trigger areas or by certain daily activities such as eating, talking, washing the face, shaving, or cleaning the teeth.

- Between paroxysms, the patient is usually entirely asymptomatic. Some patients experience a dull ache at other times.

- No neurological deficit.

- Attacks are stereotyped in the individual patient.

- Exclusion of other causes of facial pain by history, physical examination and special investigations when necessary.

A less common form of the disorder called 'Atypical Trigeminal Neuralgia' may cause less intense, constant, dull burning or aching pain, sometimes with occasional electric shock-like stabs. Both forms of the disorder most often affect one side of the face, but some patients experience pain at different times on both sides.

\section{Diagnosis}

ITN is universally considered to be one of the most painful afflictions known. Severe pain suggestive of ITN but with physical signs such as facial sensory or motor impairment can result from lesions discussed above. These serious conditions must therefore be excluded by history, examination; including neurological assessment especially of cranial nerves, and investigations; including imaging (usually MRI) to exclude space-occupying or demyelinating disease, and blood tests to exclude infections and systemic vasculitides.

Only then can the term idiopathic (benign) trigeminal neuralgia be used.

\section{Management}

Few patients with ITN have spontaneous remission and thus treatment is usually indicated. However, ITN is often an intermittent disease with apparent remissions lasting months or years but recurrence is common and very often the pain spreads to involve a wider area over time and the intervals between episodes tend to shorten.

Patients with supposed ITN are best seen at an early stage by a Specialist in order to confirm the diagnosis and initiate treatment. In the acute situation the patient's symptoms may be controlled on a short term basis with injection of a regional local anaesthetic.

Medical treatment, typically using anticonvulsants, is successful for most patients (Table 3). Carbamazepine is the main drug used, but it is not an analgesic and must be given continuously prophylactically for long periods, and under strict medical surveillance. Adverse effects must be monitored, including:

- balance (disturbed - ataxia); this tends to be the feature that limits the dose of carbamazepine

- blood pressure (may increase); patients must have a baseline test and then blood pressure estimations for three months, then six-monthly

- blood tests - mainly for liver function (may become impaired); and bone marrow function (red and white cells and/or platelets may be depressed).

\section{Table 3 Medical and surgical treatments for ITN}

\section{Medical}

Carbamazepine

Gabapentin

Phenytoin

Lamotrigine

Baclofen

Surgical

Cryotherapy

Balloon compression of trigeminal ganglion

Microvascular decompression

Gamma knife surgery 
Other agents such as gabapentin, phenytoin, lamotrigine and baclofen are available and some patients also report having reduced or relieved pain by means of alternative medical therapies such as acupuncture, chiropractic adjustment, self-hypnosis or meditation.

Should medical care become ineffective, or produce excessive undesirable side effects, neurosurgical procedures are available to relieve pressure on the nerve or to reduce nerve sensitivity.

Websites and patient information

http://www.painfoundation.org/

http://www.tna-support.org/

http://www.mayoclinic.com

\section{GLOSSOPHARYNGEAL NEURALGIA}

Glossopharyngeal neuralgia is much less common than trigeminal neuralgia. Occasionally glossopharyngeal neuralgia is secondary to tumours. The pain is of a similar nature but affects the throat and ear, and typically is triggered by swallowing or coughing. Carbamazepine is usually less effective than for trigeminal neuralgia and adequate relief of pain can be difficult. A specialist opinion is warranted to investigate and manage these patients.

\section{HERPETIC AND POST-HERPETIC NEURALGIA}

Herpes zoster (shingles), the recrudescence of herpes-varicella-zoster virus latent in sensory ganglia after chickenpox, is often preceded and accompanied by neuralgia, but a unilateral rash and ulceration is typical (Fig. 2). Neuralgia may also persist (post-herpetic neuralgia) after the rash has resolved and can cause continuous burning pain, in contrast to the lancinating pain of trigeminal neuralgia, which also affects mainly elderly patients A specialist opinion is warranted to investigate and manage these patients.

\section{PSYCHOGENIC CAUSES OF OROFACIAL PAIN}

Psychogenic (tension) headaches caused by anxiety or stress induced muscle tension are common, especially in young adults. The pain typically, affects the frontal, occipital and/or temporal regions, as a constant ache or bandlike pressure, often worse by the evening, but usually abates with rest. Similar problems can affect the orofacial region.

Reassurance may be effective but the pain may also be helped by massage, warmth, by non-steroidal anti-inflammatory drugs (NSAIDs), or by benzodiazepines - which are both anxiolytic and mild muscle relaxants, or by complementary therapies.

In some studies, nearly $40 \%$ of the population have reported frequent headaches and orofacial pain. The reason behind conditions with a psychogenic component, sometimes termed medically unexplained symptoms (MUS), may include:

- Possible links between neuro-humoural mechanisms and altered CNS function.
- The heightening of bodily sensations (lowered pain threshold) as a consequence of physiological processes such as autonomic arousal, muscle tension, hyperventilation, or inactivity.

- Misattribution of normal sensations to serious physical disorders.

Features common to most MUS include:

- Constant chronic discomfort or pain.

- Pain often of a dull boring or burning type.

- Pain poorly localised.

- Pain may cross the midline to involve the other side or may move elsewhere.

- Pain which rarely wakens the patient from sleep.

- Total lack of objective signs of organic disease.

- All investigations to identify an underlying organic illness are also negative.

- There are often recent adverse 'life events' such as bereavement or family illness.

- There are often multiple oral and/or other MUS, such as headaches, chronic back or neck pain, pruritus, irritable bowel syndrome, insomnia, numbness or dysmenorrhoea.

- Cure is uncommon in most, yet few sufferers seem to try or persist using analgesics.

Patients may bring diaries of their symptoms to emphasise their problem. Some have termed this the 'malady of small bits of paper' and though there is by no means always a psychogenic basis, such notes characterise patients with MUS. These days, this is being replaced by Internet print-outs, which are also increasingly brought by well-informed patients who have no psychogenic problems whatsoever.

Occasional patients quite deliberately induce painful oral lesions and some have Munchausen's syndrome, where they behave in such a fashion as to appear to want operative intervention.

The most common types of orofacial pain with a strong psychogenic component are:

- atypical facial pain

- oral dysaesthesia (burning mouth syndrome: BMS)

- atypical odontalgia

- the syndrome of oral complaints

- some clinicians also include temporomandibular pain-dysfunction in this category.

\section{ATYPICAL FACIAL PAIN}

Atypical facial pain (AFP) is a constant chronic orofacial discomfort or pain, defined by the International Headache Society as facial pain not fulfilling other criteria. Therefore, like burning mouth syndrome (see below), it is also a diagnosis reached only by the exclusion of organic disease; there are no physical signs, investigations are all negative and it is an MUS. Atypical facial pain is fairly common, affecting probably around $1-2 \%$ of the population. It is sometimes termed persistent idiopathic facial pain. 


Key points for dentists:
atypical facial pain
Similar symptoms may be seen in
some neurological conditions
Cranial nerve examination should be
carried out
X-rays, scans and/or blood tests are
often required

Key points for patients:
atypical facial pain
This is fairly common
The cause is not completely known
It may be caused by increased nerve
sensitivity
There may be a background of stress
There are usually no serious long-
term consequences
X-rays and blood tests may be
required
Treatment takes time and patience;
some nerve-calming drugs can help

Atypical facial pain is often of a dull boring or burning type character and ill-defined location and there is:

- a total lack of objective signs

- a negative result from all investigations

- no clear explanation as to cause

- poor response to treatment.

Patients are often middle-aged or older and $70 \%$ or more are females. Most sufferers from AFP are otherwise normal individuals who are or have been, under extreme stress such as bereavement, or concern about cancer. There are often recent adverse life-events, such as bereavement or family illness and/or dental or oral interventive procedures.

\section{Clinical features}

History findings in AFP include pain mainly in the upper jaw, of distribution unrelated to the anatomical distribution of the trigeminal nerve, poorly localised, and sometimes crossing the midline to involve the other side or moving to another site. Pain is often of a deep, dull boring or burning, chronic discomfort, and persists for most or all of the day but does not waken the patient from sleep. However the patient may report difficulty sleeping.

There may also be multiple oral and/or other psychogenic related complaints, such as dry mouth, bad or altered taste, thirst, headaches, chronic back pain, irritable bowel syndrome or dysmenorrhoea. Patients only uncommonly use analgesics to try and control the pain but there is a high level of use of health care services. There have often already been multiple consultations and attempts at treatment.

Pain is accompanied by altered behaviour, anxiety or depression. Over 50\% of such patients are depressed or hypochondriacal, and some have lost or been separated from parents in childhood. Many lack insight and will persist in blaming organic diseases (or health care professionals) for their pain.

Clinical examination is unremarkable with a total lack of objective physical (including neurological) signs. All imaging studies and blood investigations are negative.

\section{Diagnosis of AFP}

Diagnosis of atypical facial pain is clinical through careful examination of the mouth, perioral structures, and cranial nerves, and imaging (tooth/jaw/sinus radiography and MRI/CT scan) to exclude organic disease such as space-occupying or demyelinating diseases (Table 4).

\section{MANAGEMENT OF PATIENTS SUFFERING ATYPICAL FACIAL PAIN OR PAIN WITH A PSYCHOGENIC BASIS}

Few patients with AFP have spontaneous remission and thus treatment is usually indicated (Fig. 3).

Reassurance and attention to any factors such as the dentures or haematinic deficiencies may be indicated, but active dental or oral surgical treatment, or attempts at 'hormone replacement', or polypharmacy in the absence of any specific indication, should be avoided. Do not repeat examinations or investigations at subsequent appointments, since this only serves to reinforce abnormal illness behaviour and health fears.

Avoid attempts at relieving pain by operative intervention - since these are rarely successful; indeed, active dental measures such as restorative treatment, endodontics or oral surgical treatment, in the absence of any specific indication, should be avoided as they may simply reinforce the patient's perception that the pain has an organic basis.

However, it is important where possible, to identify and relieve factors which lower the pain threshold (fatigue, anxiety and depression). Simple analgesics such as NSAIDs should be tried initially, before embarking on more potent preparations.

Patient information is a very important aspect in management. Cognitive-behavioural therapy (CBT) or a specialist referral may be indicated.

It is important to clearly acknowledge the reality of the patient's symptoms and distress and never attempt to trivialise or dismiss them.

Try to explain the psychosomatic background to the problem, ascribing the symptoms to causes for which the patient cannot be blamed

Set goals which include helping the patient cope with the symptoms rather than attempting any impossible cure

Offer referral to a specialist or a trial of antidepressants, explaining that these agents are being used to treat the symptoms not depression, that some antidepressants have analgesic activity and that antidepressants have been shown in controlled trials to be effective for this problem, even in non-depressed persons.

\section{Websites and patient information}

http://facial-neuralgia.org/conditions/ atfp.html

\section{BURNING MOUTH 'SYNDROME' (BMS)}

There may be definable organic causes of this type of complaint, often described as a burning sensation (Table 5), and a patient in such pain may well also manifest psychological reactions to the experience. However, burning mouth 'syndrome' (BMS; also known as glossopyrosis; glossodynia; oral dysaesthesia; or stomatodynia) is the term usually used when symptoms described as a burning sensation, exist in the absence of identifiable organic aetiological factors. BMS is often a MUS but it must also be recognised that it may well not be a single entity.

BMS is a fairly common chronic complaint, affecting up to 0.7 to $2.6 \%$ of the population and seen especially in middle age or elderly patients, particularly in females, in a ratio of more than $3: 1$ and even as high as $7: 1$. There is no specific relationship to hormonal changes, 
despite the fact that BMS is often seen in middle aged or elderly peri- or post-menopausal females. BMS has been reported in 10-40\% of women presenting for treatment of menopausal symptoms.

Defined clinical conditions that must be excluded since they can also present with burning include:

- erythema migrans (geographic tongue)

- lichen planus

- dry mouth

- candidosis

- glossitis such as may be associated with haematinic (iron, folic acid, vitamin B) deficiency

- diabetes.

Uncommon causes that may need to be considered include:

- hypothyroidism

- lupus erythematosus

- mucositis

- drugs (especially angiotensin-converting enzyme [ACE] inhibitors; protease inhibitors; cytotoxic agents; clonazepam)

- hypersensitivity (to sodium metabisulphite, nuts, dental materials and other substances)

- galvanic reactions to metals in the mouth.

Organic problems which sometimes present with no detectable clinical lesions, but that can cause similar symptoms include:

- A haematological deficiency state (deficiencies in iron, folic acid or vitamin B) in about $30 \%$.

- Restricted tongue space from poor denture construction.

- Parafunction such as nocturnal bruxism or tongue-thrusting.

- Neuropathy - such as follows damage to the chorda tympani nerve.

No precipitating cause for BMS can be identified in over $50 \%$ of the patients but, in others, a psychogenic cause such as anxiety, depression or cancerophobia can be identified in about $20 \%$, and in some patients, BMS appears to follow either dental intervention or an upper respiratory tract infection.

\section{Clinical features}

BMS most frequently affects the tongue, but it can also affect the palate or, less commonly, the lips or lower alveolus. The history is that the burning sensation is chronic, usually bilateral, often relieved by eating and drinking, in contrast to pain caused by organic lesions which is typically aggravated by eating. Alcohol may also relieve or reduce the symptoms.

Patients with BMS often have multiple oral and/or other psychogenic related complaints, such as dry mouth, bad or altered taste, thirst, headaches, chronic back pain, irritable bowel syndrome or dysmenorrhoea. There may be changes in sleep patterns and mood and, though patients only uncommonly use analgesics to try and control the symptoms, there have often already been multiple consultations. Interestingly, patients with BMS also have heightened ability to taste - they are 'supertasters'.

Examination shows no clinically detectable signs of mucosal disease or tenderness or swelling of the tongue or affected area, and no neurological or other objective signs.

\section{Diagnosis}

Diagnosis of BMS is clinical and it is important to exclude organic causes such as erythema migrans (geographic tongue), candidosis, lichen planus, dry mouth, glossitis, diabetes or denture problems. Importantly, all investigations prove normal.

Investigations indicated, may include:

- laboratory screening for anaemia, a vitamin or iron deficiency ( blood tests)

- diabetes (blood and urine analyses)

- thyroid dysfunction (blood analyses)

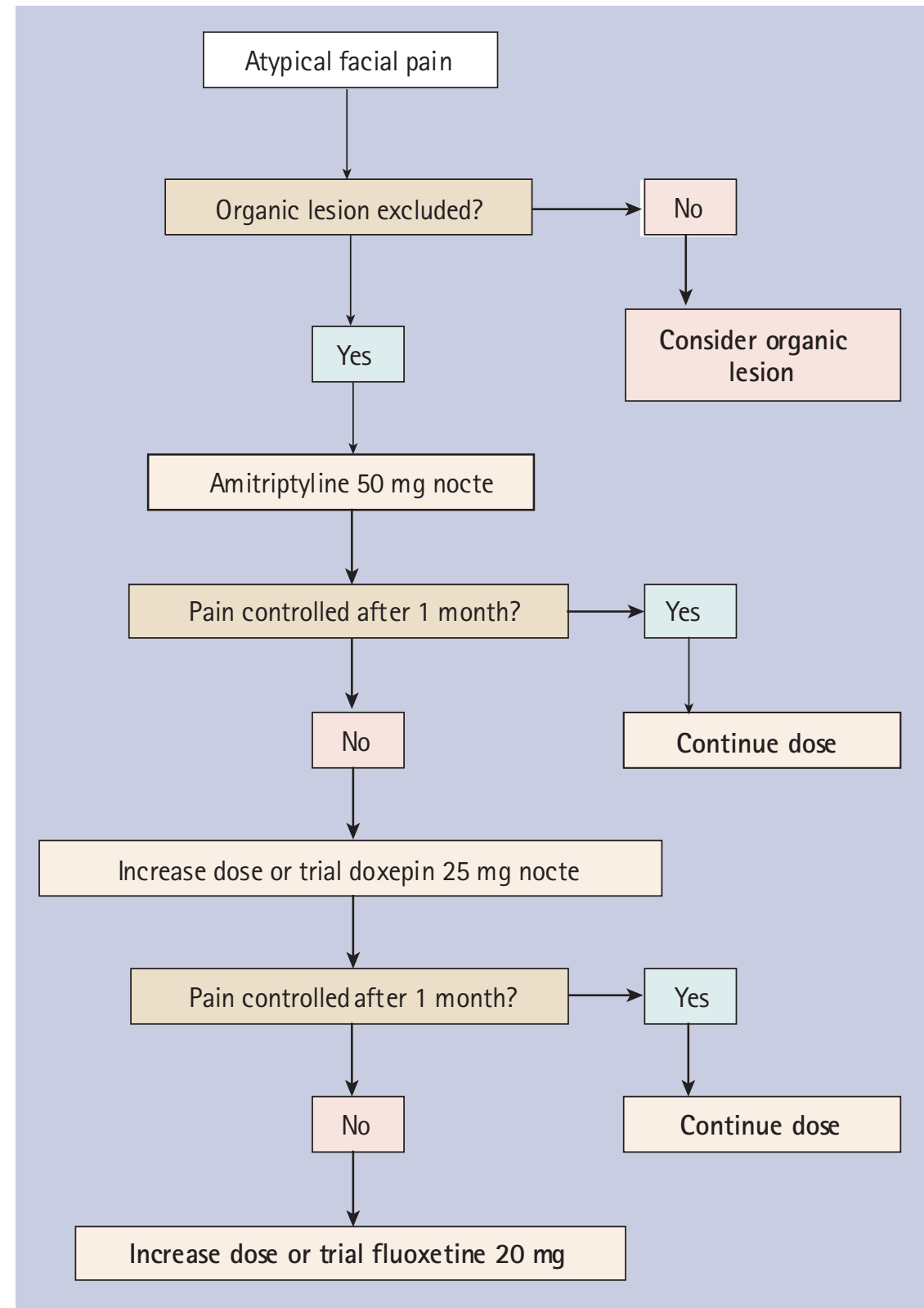

Fig. 3 Management of AFP 


\begin{tabular}{|c|c|c|c|}
\hline & $\begin{array}{l}\text { Idiopathic trigeminal } \\
\text { neuralgia }\end{array}$ & Atypical facial pain & Migrainous neuralgia \\
\hline Age (years) & $>50$ & $30-50$ & $30-50$ \\
\hline Gender & $\mathrm{F}>\mathrm{M}$ & $\mathrm{F}>\mathrm{M}$ & $M>F$ \\
\hline Site & $\begin{array}{l}\text { Unilateral, mandible } \\
\text { or maxilla }\end{array}$ & + Bilateral, maxilla & Retro-orbital \\
\hline Associated features & - & +/-Depression & $\begin{array}{l}\text { +/- Conjunctival } \\
\text { injection } \\
\text { +/- Lacrimation } \\
\text { +/- Nasal congestion }\end{array}$ \\
\hline Character & Lancinating & Dull & Boring \\
\hline Duration of episodes & Brief (seconds) & Continual & Few hours \\
\hline Usual timing of pain & Daytime & Daytime & Night time \\
\hline Precipitating factors & Trigger areas & $\begin{array}{l}+/ \text { - adverse life } \\
\text { events }\end{array}$ & + - Alcohol \\
\hline Main treatments & Carbamazepine & $\begin{array}{l}\text { Cognitive behavioural } \\
\text { therapy, } \\
\text { antidepressants }\end{array}$ & $\begin{array}{l}\text { Oxygen, } \\
\text { sumatriptan }\end{array}$ \\
\hline
\end{tabular}

Keypoints for dentists: Burning mouth syndrome Similar symptoms may be seen in some organic conditions Blood tests may be required Psychological assessment can be helpful

Keypoints for patients: Burning mouth syndrome This is a common condition The cause is not usually known It may be a nerve hypersensitivity It is not infectious

It may occasionally be caused by some mouth conditions, dry mouth, deficiencies, diabetes or drugs It has no long-term consequences Blood tests or biopsy may be required It may be controlled by some nervecalming drugs
- xerostomia (salivary flow rates)

- candidosis (oral rinse).

- psychological screening using, for example, the Hospital Anxiety and Depression (HAD) scale.

Management is discussed above as for AFP.

\section{Websites and patient information}

http://www.mssm.edu/msjournal/65/05_miy.pdf http://www.go4hope.org

http://www.mayoclinic.com

\section{ATYPICAL ODONTALGIA}

Atypical odontalgia is pain and hypersensitive teeth in the absence of detectable pathology. The pain is typically indistinguishable from pulpitis or periodontitis but is aggravated by dental intervention. Probably a variant of atypical facial pain, it should be managed similarly.

\section{The syndrome of oral complaints}

Multiple pains and other complaints may occur simultaneously or sequentially, and relief is rarely found (or admitted). may bring diaries of their symptoms to emphasise their problem.

\section{VASCULAR CAUSES OF OROFACIAL PAIN}

Several disorders in which the most obvious organic feature is vascular dilatation or constriction can cause orofacial pain. The pain is usually obviously in the face or head rather than in the mouth alone but occasionally can involve both, and can be difficult to differentiate from other causes of orofacial pain. These disorders include:

- Migraine (usually obvious and not causing oral pain alone, and therefore not included here).

- Migrainous neuralgia.

- Giant cell arteritis.

- Migrainous neuralgia (cluster headache).

Migrainous neuralgia is less common than migraine but more likely to cause orofacial pain.
Males are mainly affected and attacks often begin about middle age (Table 5). The pain is unilateral, occurs in attacks, is burning and 'boring' in character, and localised around the eye usually. Generally, the attacks commence, and often awaken the patient, at the same time each night or in the early hours of the morning - hence the term 'alarm clock headache'. This pain may be associated with profuse watering and 'congestion' of the conjunctiva, rhinorrhoea and nasal obstruction on the affected side. The attacks usually end in less than one hour. Attacks are sometimes precipitated by alcohol.

Migrainous neuralgia is managed by a specialist, with a variety of agents, including sumatriptan, beta-blockers, indometacin, or oxygen inhalations.

\section{CRANIAL ARTERITIS (TEMPORAL ARTERITIS; GIANT-CELL ARTERITIS)}

Cranial arteritis is a febrile disease, in which giant cells appear in the arteries and cause a deranged internal elastic lamina. It most commonly affects the elderly.

The headache is intense, deep and aching, throbbing in nature and persistent. It is frequently made worse when the patient lies flat in bed and it may be exacerbated or reduced by digital pressure on the artery involved. Occasionally the artery (usually the superficial temporal artery) may be enlarged and tender. It is also characterised by malaise, weakness, weight loss, anorexia, fever, and sweating.

Diagnosis is supported by a raised erythrocyte sedimentation rate (or plasma viscosity). Arterial biopsy demonstrates fragmentation of the internal elastic lamina.

Although it is a self-limiting disease, patients with cranial arteritis may be threatened with loss of vision, and therefore need urgent diagnosis and treatment by a specialist: a systemic corticosteroid (prednisolone) is indicated.

\section{REFERRED CAUSES OF OROFACIAL PAIN}

Pain may occasionally be referred to the mouth, face or jaws from the:

- Neck: cervical vertebral disease, especially

Table 5 Causes of a burning sensation in the mouth

Local causes

Erythema migrans (geographical tongue)

Lichen planus

Candidosis

Denture problems

Parafunctional activity (eg tongue thrusting habit, clenching)

Systemic causes

Psychogenic

Cancerophobia

Depression

Anxiety states

Hypochondriasis

Deficiency of: Vitamin B, especially B12; Folate; Iron

Dry mouth

Diabetes

Drugs 
cervical spondylosis, very occasionally causes pain referred to the face.

- Heart in patients with angina. The pain usually affects the mandible, is initiated by exercise (especially in the cold) and abates quickly on rest.

- Lungs: orofacial pain emanating from lung cancer is a well-recognised entity and can mimic for example, TMJ pain-dysfunction syndrome.

- Oesophagus; pain plus sialorrhoea may result from oesophageal lesions.

- Styloid process; Eagle's syndrome, a rare disorder due to an elongated styloid process (stylalgia), may cause pain on chewing, swallow- ing or turning the head.

- Eyes: pain from the eyes, arising for example, from disorders of refraction, retrobulbar neuritis (eg in multiple sclerosis), or glaucoma, can radiate to the orbit, maxilla or frontal region.

- Ears: middle ear disease may cause headaches or pain in the TMJ region. Conversely, oral disease not infrequently causes pain referred to the ear, particularly from lesions of the posterior tongue.

- Pharynx; carcinoma of the pharynx may cause orofacial pain.

A specialist opinion is warranted to investigate and manage these patients.

\section{Patients to refer}

Trigeminal neuralgia in view of possibility of demyelination or space occupying lesion

Giant cell arteritis in view of risk of blindness

Patients with atypical facial pain who need psychological help Malignancy 